\title{
Influence of Crystal Orientations on the Bendability of an Al-Mg-Si Alloy
}

\author{
Hiroki Takeda ${ }^{1}$, Akira Hibino ${ }^{1}$ and Ken Takata ${ }^{2}$ \\ ${ }^{1}$ Technical Research Division, Furukawa-Sky Aluminum Corporation, Fukaya 366-8511, Japan \\ ${ }^{2}$ Technical Development Bureau, Nippon Steel Corporation, Futtsu 293-8511, Japan
}

\begin{abstract}
Aluminum alloy sheets are being increasingly used to reduce the weight of an automotive body. Al-Mg-Si alloys have been used because they have favorable properties for automotive bodies. One of the important requirements of these alloys is the ability to resist fracture while bending. In this study, the influence of crystal orientations on the bendability of an Al-Mg-Si alloy is investigated using single-crystal specimens from the viewpoint of the shear band formation. The bendability and the formation of shear bands are clearly dependent on the crystal orientation, and $\langle 001\rangle / /$ ND-oriented specimens exhibit the highest bendability. The formation of denser shear bands is observed with a decrease in bendability. The shear bands behaved as the origin of the cracks and the propagation path. It is concluded that the inhibition of the formation of shear bands by controlling the orientations is very important to improve the bendability. Moreover, there exists a relation between the Taylor factor and the bendability because the bendability tended to decrease with an increase in the Taylor factor.
\end{abstract} [doi:10.2320/matertrans.L-MG200951]

(Received October 2, 2009; Accepted December 25, 2009; Published March 25, 2010)

Keywords: bendability, aluminum-magnesium-silicon, single crystal, crystal orientations, shear bands

\section{Introduction}

Aluminum alloys are increasingly being used to manufacture lightweight automotive bodies. Al-Mg-Si alloys have been used because they have favorable properties for automotive bodies. High bendability of an aluminum sheet is one of the desirable properties when it is applied to the fabrication of an automotive body sheet. Thus far, there have been some studies on the bendability of aluminum alloys. Alloys having high yield strength exhibit low bendability. ${ }^{1,2)}$ Precipitates on grain boundaries, secondphase particles, and a formation of shear bands upon bending decrease bendability. ${ }^{1-3)}$ The texture of the alloy affects the formation of the shear bands upon bending. ${ }^{4,5}$ ) These studies have been carried out with polycrystals of aluminum alloys, and the relation between the crystal orientation of the alloy and its bendability has not yet been clearly understood.

In this study, the relation between the crystal orientation of an Al-Mg-Si alloy and its bendability has been investigated using single-crystal specimens from the viewpoint of the formation of shear bands upon bending.

\section{Experimental}

Table 1 shows the chemical composition of the specimens used in this study. In order to suppress the influence of second-phase particles on bendability and obtain large grains for the single-crystal specimens, the $\mathrm{Fe}, \mathrm{Mn}$, and $\mathrm{Cr}$ contents were reduced as compared to commercial Al-Mg-Si alloys.

An ingot (length: $700 \mathrm{~mm}$; width: $200 \mathrm{~mm}$; thickness: $80 \mathrm{~mm}$ ) was prepared by using the direct chill (DC) casting method in the laboratory. After homogenization, this ingot was hot-rolled, cold-rolled, annealed, and cold-rolled again. Then, a 1.0-mm-thick sheet was obtained. This sheet was solution-treated at a temperature of $843 \mathrm{~K}$ for $18 \mathrm{ks}$. After this treatment, the sheet was quenched in water and aged at a temperature of $373 \mathrm{~K}$ for $72 \mathrm{ks}$ (under-age condition) to achieve precipitation hardening because the low yield
Table 1 Chemical composition of the alloy (mass\%).

\begin{tabular}{ccccccccc}
\hline $\mathrm{Cu}$ & $\mathrm{Si}$ & $\mathrm{Fe}$ & $\mathrm{Mn}$ & $\mathrm{Mg}$ & $\mathrm{Zn}$ & $\mathrm{Cr}$ & $\mathrm{Ti}$ & $\mathrm{Al}$ \\
\hline 0.0002 & 0.9969 & 0.0459 & 0.0009 & 0.5905 & 0.0047 & 0.0009 & 0.0007 & $\mathrm{Bal}$. \\
\hline
\end{tabular}

Table 2 Orientations of specimens used for the bending test.

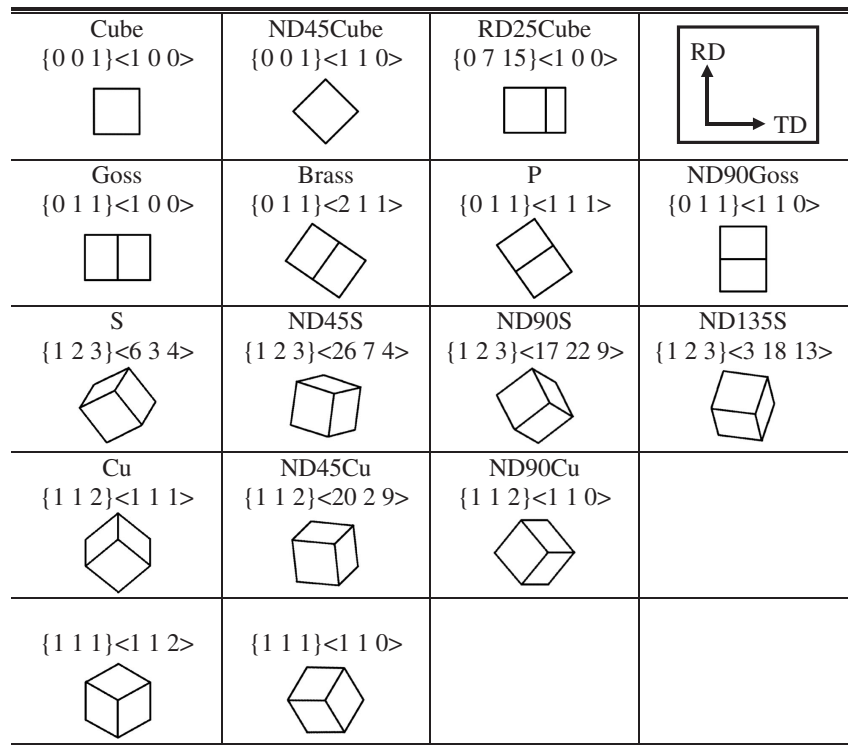

strength of the as-quenched sheet was not sufficient for distinguishing the difference in bendability. ${ }^{1,2)}$

The large grains of the sheet were observed after macroetching with the reagent (hydrochloric acid : nitric acid: water $=1: 1: 1$ ). The orientations of the grains were defined by Scanning Electron Microscope-Electron Backscatter Diffraction (SEM-EBSD). The single-crystal specimens (length: $20 \mathrm{~mm}$; width: $10 \mathrm{~mm}$ ) for bending test were cut out from these large grains having a diameter of several centimeters. The crystal orientations of these specimens are listed in Table 2. Some of the crystal orientations here were 


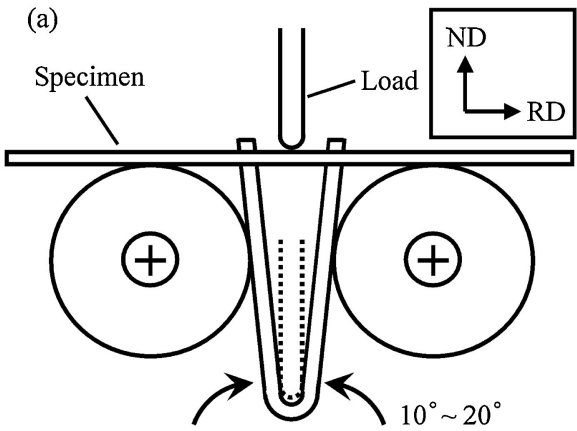

(b)

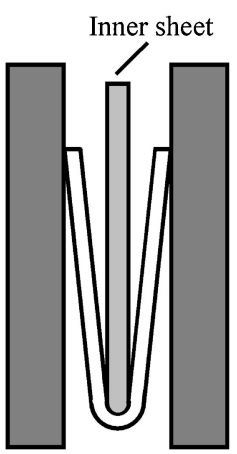

(c)

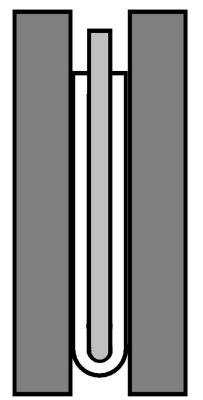

Fig. 1 Schematic representation of the bending test. Bending tests are performed in the following order: (a) bending by load, (b) inserting an inner plate between the bent plates, and (c) pressing.

typically observed in rolled or annealed FCC materials, ${ }^{6,7)}$ and the others were obtained by rotating these orientations around the nominal direction (ND) or rolling direction (RD). The crystal direction of the specimens was parallel to the RD, as listed in this table. The name of the orientations that rotated around ND or RD was defined as the combination of the rotation angle and its initial orientation hereafter. For example, the specimen defined as ND45Cube represents an orientation with a $45^{\circ}$ rotation around ND from the cube orientation. The orientation deviation of the specimens was within $\pm 10^{\circ}$.

Figure 1 shows the schematic representation of the bending test. First, a specimen on two rolls was bent by a load to form a " $v$ " shape. Then, an inner plate was inserted between the bent plates. Finally, the specimen was pressed to obtain a $180^{\circ}$ bend angle (bending radius: $0.5 \mathrm{~mm}$ ). This is called $180^{\circ}$ bending (A-bending). Some specimens were bent without an inner plate to investigate the difference among specimens with high bendabilities (B-bending). Here, the bending edge was vertical to the longitudinal direction of the single-crystal specimens that were parallel to the RD.

The bent specimens were visually graded according to their bendability on a scale of 1 to 10 . The samples are shown in Fig. 2. A score of 10 indicated that there were no cracks and no roughness on the surface of the bent specimen; on the other hand, a score of 2 indicated that there were microcracks propagating through the thickness and a score of 1 indicated that the bending specimen fractured to two pieces before reaching $180^{\circ}$ bend angle during the bending test. The bending score was defined as the average value obtained after three or more trials.
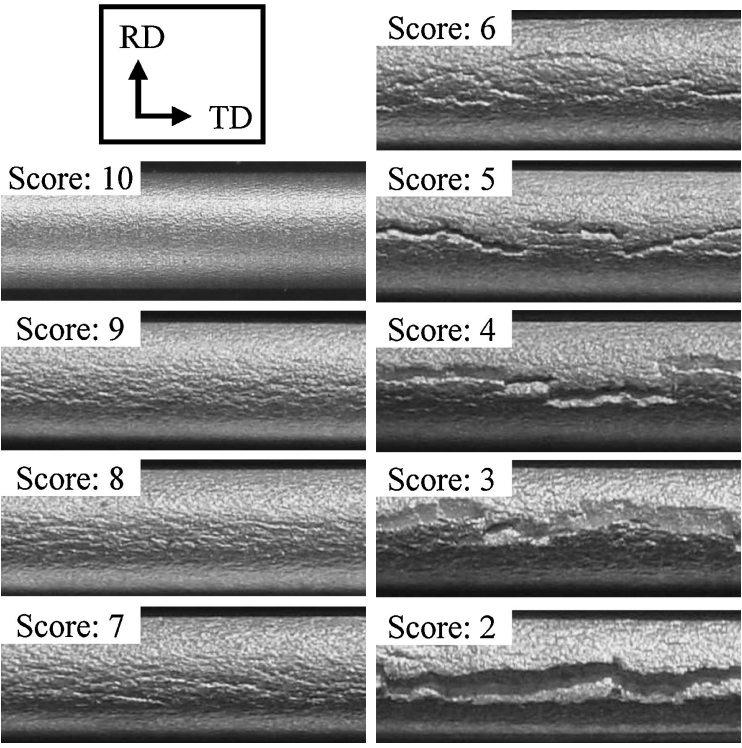

Fig. 2 Appearances of bending samples for rating bendability on a scale of 1 to 10 visually. RD and TD denote directions of samples. In the case of a score of 10 , there are no cracks and no roughness. In the case of a score of 2 , microcracks propagate through the thickness. In the case of a score of 1 , bending specimen fractured to two pieces before reaching $180^{\circ}$ bend angle.

Shear bands on bending were observed from a transverse direction (TD) cross section using an optical microscope after aging the bent specimen at $493 \mathrm{~K}$ for $1.2 \mathrm{ks}$. It becomes easy to observe shear bands by etching $\mathrm{Mg}_{2} \mathrm{Si}$ precipitates formed on the sites of the shear bands during the aging.

The Taylor factor was calculated for each orientation of the specimens using TSL OIM Analysis software (TSL Co. Ltd.), under the assumption that the bending was a plane strain deformation. Shear bands and dislocation microstructures formed upon bending were also observed from the TD cross section by Transmission Electron Microscope (TEM).

\section{Results and Discussion}

\subsection{Influence of crystal orientation on bendability}

The orientations of the specimens and their bending scores are listed in Table 3. In this table, there are Cube, Goss, S, $\mathrm{Cu},\{111\}\langle 112\rangle$ and the others that were formed by rotation around ND or RD from these orientations. Figure 3 shows the appearances of some specimens after the A-bending test and their bending scores. Cube-, ND45Cube-, and RD25Cubeoriented specimens exhibit the highest bendability. This result is in good agreement with the reports on polycrystal specimens. ${ }^{4,5)}$ Cube-, ND45Cube-, and RD25Cube-oriented specimens have similar scores of around 10 on the A-bending test; however, the Cube and RD25Cube orientations exhibit a slightly higher bendability than the ND45Cube orientation in the B-bending test. $\{111\}\langle 112\rangle-$, ND45S-, and ND90Cuoriented specimens exhibit good bendability, second only to that of the Cube, ND45Cube and RD25Cube orientations. These three orientations also have similar scores of around 9; however, the $\{111\}\langle 112\rangle$ orientation exhibits the highest bendability among the three, and ND45S orientation exhibits a higher bendability than the ND90Cu orientation in the B- 
Table 3 Relation between the orientation of the specimen and bendability. A-bending is $180^{\circ}$ bending with an inner plate, and B-bending is severe $180^{\circ}$ bending without an inner plate.

\begin{tabular}{ccc}
\hline \multirow{2}{*}{$\begin{array}{c}\text { Specimens } \\
\text { orientation }\end{array}$} & \multicolumn{2}{c}{ Bending score } \\
\cline { 2 - 3 } Cube & A-bending & B-bending \\
\hline ND45Cube & 10.0 & 10.0 \\
\hline RD25Cube & 9.0 & 9.0 \\
\hline Goss & 7.0 & 9.7 \\
\hline Brass & 4.3 & \\
\hline $\mathrm{P}$ & 3.3 & \\
\hline ND90Goss & 1.0 & \\
\hline $\mathrm{S}$ & 4.5 & \\
\hline $\mathrm{ND} 45 \mathrm{~S}$ & 8.6 & \\
\hline $\mathrm{ND} 90 \mathrm{~S}$ & 4.0 \\
\hline $\mathrm{ND} 135 \mathrm{~S}$ & 2.8 \\
\hline $\mathrm{Cu}$ & 3.3 & 6.3 \\
\hline $\mathrm{ND} 45 \mathrm{Cu}$ & 4.3 \\
\hline $\mathrm{ND} 90 \mathrm{Cu}$ & 8.8 & 7.8 \\
\hline$\{111\}\langle 112\rangle$ & 9.0 \\
\hline$\{111\}\langle 110\rangle$ & 5.4 \\
\hline
\end{tabular}

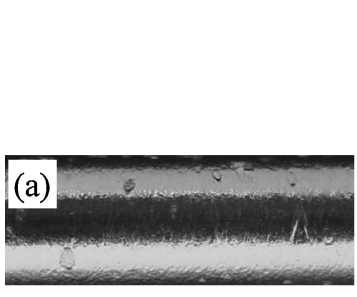

Cube : 10.0

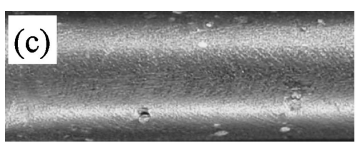

ND45S : 8.6

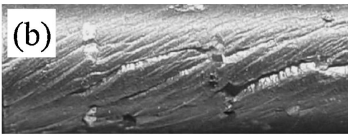

Brass : 4.3

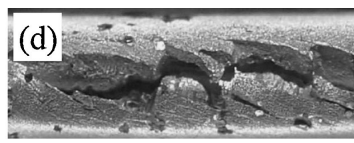

ND135S : 2.8

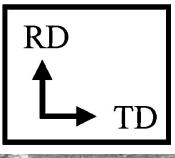

Fig. 3 Appearances of specimens after A-bending. Values denote bending scores.

bending test. On the other hand, ND135S- and ND90Gossoriented specimens exhibit very low bendability. It is revealed that the bendability is dependent on the crystal orientation because the other factors that influence the bendability, such as solid solution, fine precipitates and coarse second-phase particles are almost the same due to the same process. Although effect of crystal orientations on yield strength can not be ignored for bending, this can be still considered to originate the factor of crystal orientations themselves actually.

In order to understand the anisotropy of bendability due to the rotation of ND, five directions- $\langle 001\rangle / / \mathrm{ND}$, $\langle 011\rangle / / \mathrm{ND},\langle 123\rangle / / \mathrm{ND},\langle 112\rangle / / \mathrm{ND}$, and $\langle 111\rangle / / \mathrm{ND}-$ were chosen in this study. In each direction, there is a group of orientations with rotation around ND. Table 4 and Fig. 4 show these orientations and bendabilities in each group upon
Table 4 Classification of the specimens' orientation. The orientations of a specimen are classified into five groups in the ND direction, and each group has several orientations of specimens. Values denote bending scores (A-bending) in Table 3.

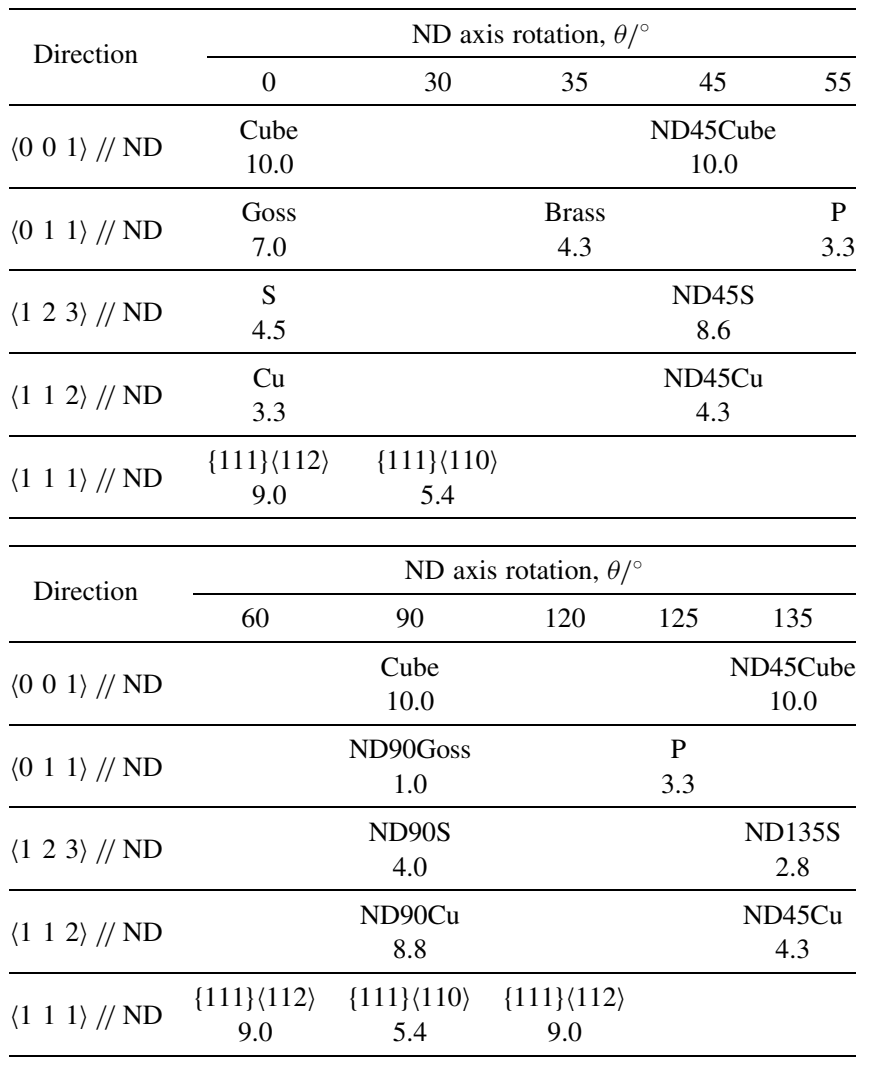

\begin{tabular}{|c|c|c|c|}
\hline \multirow{2}{*}{ Direction } & \multicolumn{3}{|c|}{$\mathrm{ND}$ axis rotation, $\theta /^{\circ}$} \\
\hline & 145 & 150 & 180 \\
\hline$\left\langle\begin{array}{lll}0 & 0 & 1\end{array}\right\rangle / / \mathrm{ND}$ & & & $\begin{array}{c}\text { Cube } \\
10.0\end{array}$ \\
\hline$\left\langle\begin{array}{lll}0 & 1 & 1\end{array}\right\rangle / / \mathrm{ND}$ & $\begin{array}{c}\text { Brass } \\
4.3\end{array}$ & & $\begin{array}{c}\text { Goss } \\
7.0\end{array}$ \\
\hline$\left\langle\begin{array}{lll}1 & 2 & 3\end{array} / / / \mathrm{ND}\right.$ & & & $\begin{array}{c}\mathrm{S} \\
4.5\end{array}$ \\
\hline$\left\langle\begin{array}{lll}1 & 1 & 2\end{array}\right\rangle / / \mathrm{ND}$ & & & $\begin{array}{l}\mathrm{Cu} \\
3.3\end{array}$ \\
\hline$\left\langle\begin{array}{lll}1 & 1 & 1\end{array}\right\rangle / / \mathrm{ND}$ & & $\begin{array}{c}\{111\}\langle 110\rangle \\
5.4\end{array}$ & $\begin{array}{c}\{111\}\langle 112\rangle \\
9.0\end{array}$ \\
\hline
\end{tabular}

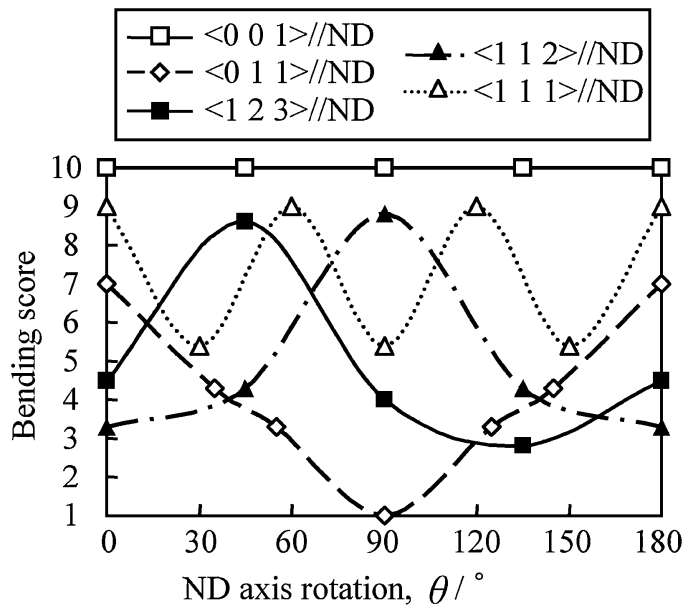

Fig. 4 Dependence of bendability in each direction on ND axis rotation. 


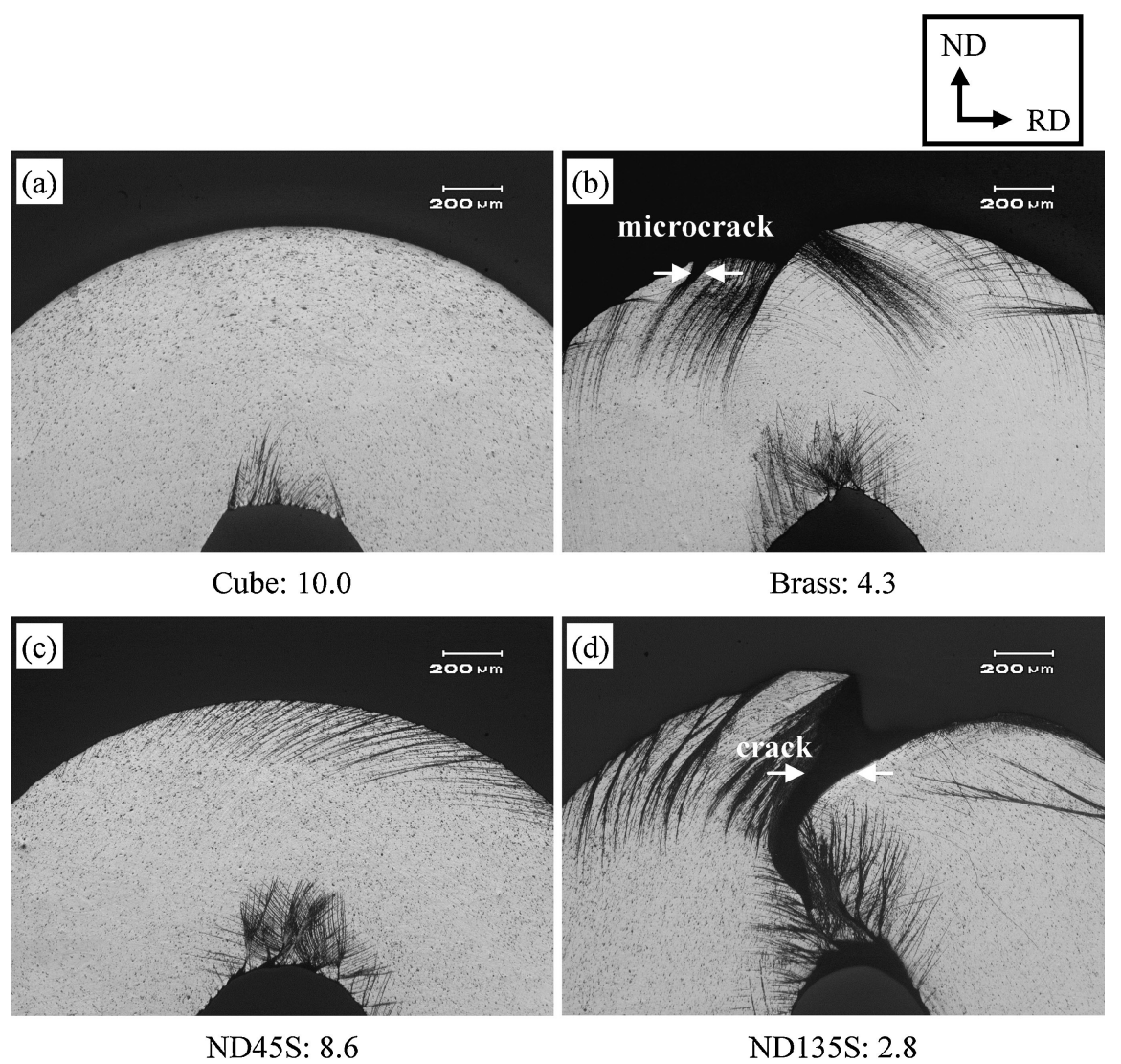

Fig. 5 Optical micrographs of shear bands after the A-bending test. Black lines and the values denote shear bands and bending scores, respectively.

ND rotation. The orientations of Cube and ND45Cube that belong to $\langle 001\rangle / /$ ND exhibit the highest score and almost no anisotropy of bendability. On the other hand, in the $\langle 011\rangle / /$ ND group, the bendability has the lowest score upon the $90^{\circ}$ rotation of ND, and the difference in bendability between the Goss and the ND90Goss orientations is very large. Therefore, there is considerable anisotropy of bendability in this group. Similarly, substantial anisotropy of bendability can be also recognized in the $\langle 123\rangle / / \mathrm{ND}$ and $\langle 112\rangle / / \mathrm{ND}$ groups. In addition, the bendability and its anisotropy of orientations in the $\langle 111\rangle / /$ ND group are relatively excellent as compared to those in the $\langle 011\rangle / / \mathrm{ND},\langle 123\rangle / / \mathrm{ND}$, and $\langle 112\rangle / / \mathrm{ND}$ groups. Finally, it could be considered that the development of the orientations in the $\langle 001\rangle / / \mathrm{ND}$ group effectively improves the bendability and reduces the anisotropy.

\subsection{Shear band formation}

Bendability is dependent on the crystal orientation. It is reported that bendability is also related to the formation of shear bands upon bending. ${ }^{1,3,4)}$ Figure 5 shows the optical micrographs of some specimens obtained after the A-bending test. The differences in the formation of shear bands can be recognized clearly on the TD cross section from these micrographs. In the Cube orientation that has the highest bendability, no shear band can be visually observed, while in the case of ND45S, some shear bands can be observed. Further, in the case of the Brass and ND135S orientations, a high density of shear bands can be observed. The micrograph of Brass orientation shows that shear bands cause the initial microcrack. One of the ND135S specimens shows that a crack propagate along the shear bands through the thickness. Therefore, it is concluded that the inhibition of the formation of shear bands by controlling the orientations is very important to the improvement of bendability.

In addition, shear bands were also observed in detail by TEM because the microstructure of the shear bands generated by bending has not been reported in a single crystal. The results of the observation in the case of ND90Goss are shown in Fig. 6. In Fig. 6(a), an approximately $1-\mu \mathrm{m}$-thick white band is denoted as the shear band. The diffraction patterns suggest that the orientations within the shear band are similar. Furthermore, a slight orientation rotation between the shear band and the matrix can be considered from their diffraction patterns. These results are not in agreement with a report on the polycrystal of austenite stainless steel deformed by rolling. ${ }^{8)}$ It appears that the microstructure formation of shear bands in single-crystal alloys is different from that in polycrystal materials. A detailed investigation of the shear bands is required in the future.

\subsection{Influence of Taylor factor on bendability}

Figure 7 shows the relation between the calculated Taylor factor and the bending score (A-bending). This figure indicates that the bending score decreases with an increase in the value of the Taylor factor. This implies that the bendability can be roughly predicted by calculating the 

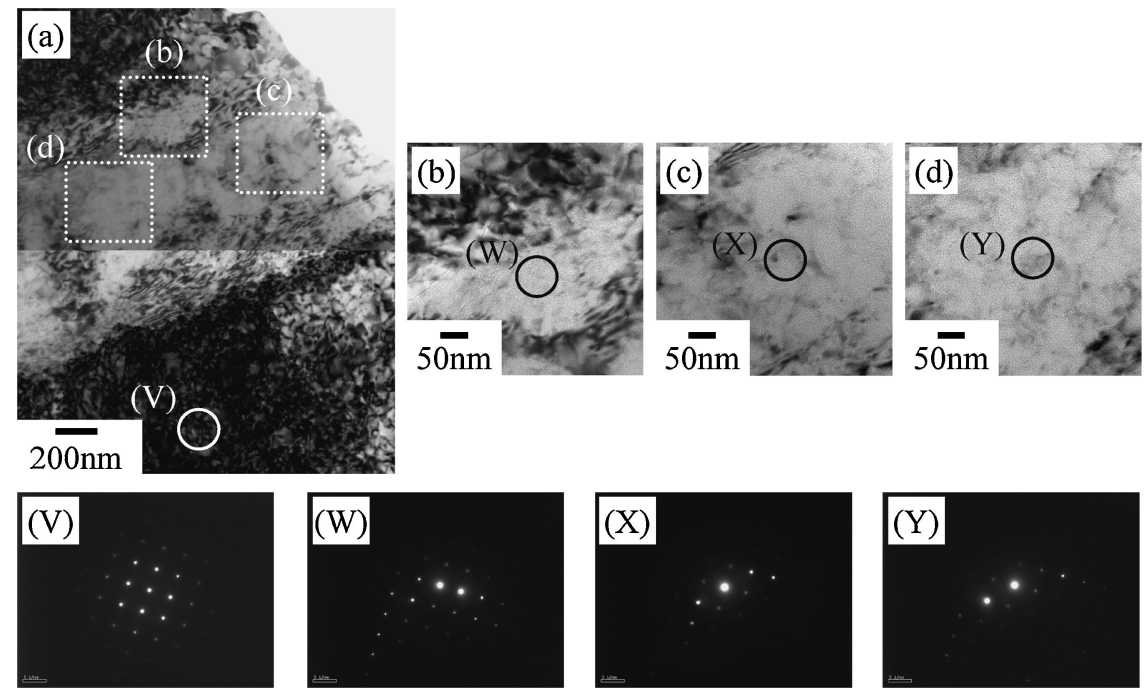

Fig. 6 TD cross-sectional TEM images of the shear band in ND90Goss from [001]Al zone axis. The specimen used for the observation was bent to an angle of $90^{\circ}$ (bending radius: $0.5 \mathrm{~mm}$ ). (a) is the outline view of the shear band. (b), (c), and (d) are the magnified micrographs of the dotted-line regions in (a). (V) indicates the diffraction pattern of the solid line region in the matrix. (W), (X), and (Y) show the diffraction pattern of the solid line region in the shear band.

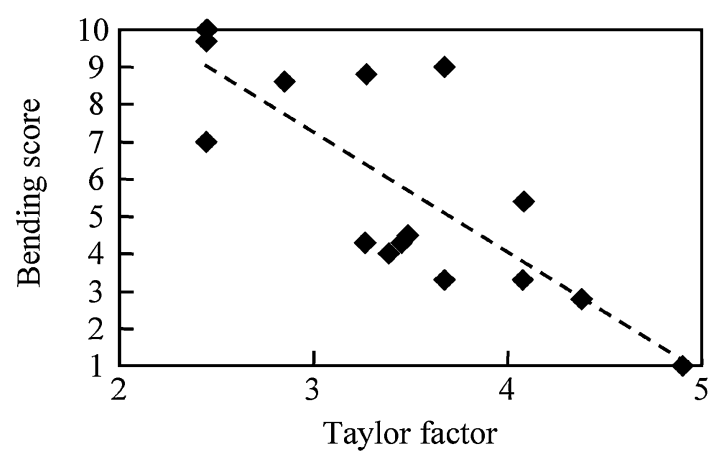

Fig. 7 Relation between Taylor factor and bending score (A-bending).

Taylor factor. The Taylor factor is closely related to the slip behavior in materials. It can be considered that a low Taylor factor leads to a relative uniform slip deformation during bending. On the other hand, if the Taylor factor is high, the necessary stress for deformation increases greatly, and a uniform slip deformation becomes difficult. Therefore, shear bands are formed as a result of relaxing further deformation.

Figure 8 shows the TEM micrographs of the bent portion of the specimens in the Cube and ND90Goss orientations. The dislocation density of the ND90Goss-oriented specimen is obviously higher than that of the Cube one. It is suggested that the large accumulation of dislocations during bending under a high Taylor factor condition leads to the easy formation of shear bands.

\section{Conclusion}

Several types of single-crystal specimens of an Al-Mg-Si alloy were produced in the laboratory and the influence of the crystal orientation on the bendability was investigated experimentally. The findings were as follows:

(1) There is a close relation between the bendability and the crystal orientation. $\langle 001\rangle / /$ ND-oriented specimens ex-

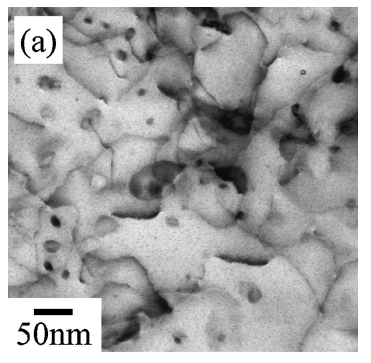

Cube : 10.0, 2.45

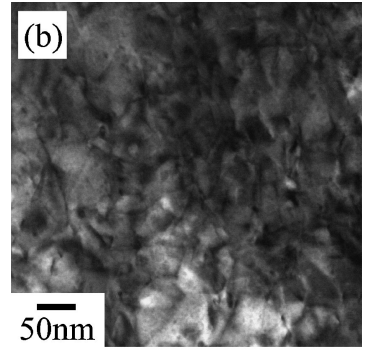

ND90Goss : $1.0,4.90$
Fig. 8 Dislocation structures in TEM micrographs of Cube and ND90Goss. Observations were carried out after $90^{\circ}$ bending (bending radius: $0.5 \mathrm{~mm}$ ). The values represent the bending score and the Taylor factor, respectively.

hibit the highest bendability and the lowest anisotropy of bendability among all types of crystal directions investigated.

(2) The formation of shear bands is dependent on the crystal orientation. The inhibition of shear bands leads to the improvement of bendability because the shear bands cause the initial microcrack and its propagation path.

(3) There also exists a relation between the bendability and the Taylor factor. The bendability improves with a decrease in the Taylor factor. The dependence of the crystal orientation on bendability can be expressed in terms of the Taylor factor.

\section{Acknowledgments}

The authors would like to express their sincere gratitude to Mr. T. Hasimoto for assisting with the experimental work. The authors also appreciate the support and encouragement of Mr. Y. Betsuki. Further, the comments and suggestions of Dr. Hirofumi Inoue (Osaka Prefecture University) led to significant improvements in this study. 


\section{REFERENCES}

1) A. Hibino, T. Muramatsu, M. Saga and K. Takata: J. JILM 53 (2003) 534-541.

2) M. Saga, M. Kikuchi, Y. Zhu and M. Matsuo: Proc. 6th Int. Conf. On Aluminum Alloys (1998) pp. 425-430.

3) M. Asano, T. Minoda, Y. Ozeki and H. Yoshida: Mater. Sci. Forum 519_
521 (2006) 771-776.

4) A. Hibino, T. Muramatsu, T. Komatsubara, M. Saga and K. Takata: Abstracts of the 106th Conference of the Japan Institute of Light Metals (2004) pp. 89-90.

5) M. Kuroda and V. Tvergaard: Int. J. Plasticity 23 (2007) 244-272.

6) J. Hirsch and K. Lücke: Acta Metall. 36 (1988) 2863-2882.

7) J. Hirsch and K. Lücke: Acta Metall. 36 (1988) 2883-2904.

8) K. Higashida and T. Morikawa: Tetsu-to-Hagané 94 (2008) 576-571. 